\title{
Sur Guillaume Fabri, de Hilden, sa famille et sa femme, quelques renseignements nouveaux
}

\author{
Par Eugène Olivier, Le Mont-sur-Lausanne
}

N'est-ce que vaine curiosité, ce désir que nous éprouvons de connaître par le menu les conditions de vie des hommes qui ont fait progresser notre art, de revivre avec leur personne, de nous plonger dans l'entourage qui fut le leur? J'y vois plutôt un besoin de sympathie, de contact direct, qui, nous rendant l'homme plus proche, nous fera mieux apprécier l'œuvre. C'est dans ce sens que je voudrais voir interpréter les quelques apports que voici offerts à la mémoire du bon maître rhénan, dont la carrière se fit surtout à Lausanne, Payerne et Berne. Il n'a pas seulement été l'un des chefs incontestés de la chirurgie de son siècle, le Machaon de son époque comme le déclarait un de ses admirateurs lausannois; nous aurions tort de le tenir pour mort puisque les médecins d'aujourd'hui continuent, sur divers points, à appliquer des règles qu'il fut le premier à poser et qui ont chance de rester valables pour toujours.

Grâce aux centaines d'observations que le laborieux chirurgien date et situe exactement, le cadre, tantôt mouvant, tantôt stable, où s'est déroulée sa longue carrière, peut se reconstituer sans trop de lacunes. Pour sa femme aussi, Marie Colinet - on disait en son temps Colinette -, il aime à mettre en relief ses interventions thérapeutiques heureuses. La naissance de quelques uns de leurs enfants, parfois leur mort, sont mentionnées par lui. Là déjà, cependant, il s'en tient le plus souvent à une ligne ou deux; et lorsqu'il s'agit des membres de sa famille, père, mère, frère, sœur, son laconisme s'accentue encore. Ainsi, sur le milieu familial qui fut celui de son enfance, les écrits de l'homme fait ne nous livrent que quelques miettes; et qui restent miettes même avec l'apport des archives locales.

De son père, tout ce qu'il nous dit est que Petrus Andreas Fabricius était greffier du tribunal de Hilden au moment de sa mort, en 1569, laissant veuve et trois orphelins ${ }^{1}$. De sa mère, qu'en mars 1612 elle approchait des quatre-vingts ans et était gravement malade, hydropique ${ }^{2}$; elle devait être une AUfF Der Koulen, son frère Theodore apparaissant à diverses re-

\footnotetext{
${ }^{1}$ Spiegel deß menschlichen Lebens (1621), Vorrede. - Ce passage, le seul où FABRI énumère tous les membres de sa famille, semble avoir échappé jusqu'ici à ses biographes.

${ }^{2}$ Opera Omnia (0.0.) 1020.
} 
prises dans les observations de FABRICE $^{3}$. Et qu'elle eut trois enfants: luimême, Guillaume, né le 25 juin $1560^{4}$; un second garçon, dont nous ignorons le nom et la date exacte de la naissance, FABRI le disant tantôt né vers $1562^{1}$, tantôt vers $1565^{5}$; il mourut le 28 janvier 1581, de calculs urinaires, attribués par son médecin à ce qu'il salait à l'excès tout ce qu'il mangeait. Une fillette, enfin, qui avait un an à la mort du père ${ }^{1}$.

A ce très modeste noyau, les documents d'archives ont apporté des compléments bienvenus ${ }^{6}$. Les dates précises du décès des parents: le père, 6 novembre 1569; la mère, 26 avril 1612. Son prénom, Marg UERITE. Elle s'était assez tôt remariée à Peter Crantz, un brave homme, mais qui ne tarda pas à mourir aussi, ou bien ne réussit pas à gagner de quoi faire étudier Guillaume au delà de sa treizième année. Tout ce que celui-ci apprendra, c'est à sa vaillance et à sa propre initiative qu'il le devra. La sœur, enfin, avait pour nom Gertgen. - Petite récolte, on le voit, et assez surprenante à certains égards; car les papiers officiels nomment la mère MARgarete AUFF DEM SAND; montrant que là, comme chez nous à certaines époques, florissait la coutume des noms de rechange, des «alias». Pour le père, même, on est conduit à conclure qu'il ne devait pas être connu sous le nom de Fabricius - que lui donnent son fils et l'annaliste Teschenmagher - ou de Fabri, que le chirurgien porta de son vivant. Ce nom semble ne pas exister dans la région; il n'aura été que la latinisation d'un Sснмidт ou Sснмітz. Nom incertain, origine modeste, études scolaires quasi inexistantes, pareil début promettait peu, à vues humaines; d'autant plus d'honneur à celui qui tira de ces éléments la carrière que nous savons.

Voici maintenant une pièce, apparemment inconnue, que nous ajoutons à ce dossier si sommaire; elle n'est autre que le sermon prononcé aux funérailles de la mère du grand chirurgien. Et par le propre gendre de la défunte, le beau-frère de Guillaume, l'époux, donc, de cette Gertgen dont jusqu'ici l'on savait si peu: «Leichpredig bey der Begräbnuss der Ehrentreichen und Tugentsamen Frawen Margaretha vom Sandt, gewesener Ehe-

${ }^{3}$ O.O. 19, 29, 49, 84; avunculus meus, Theodorus AUfF DER Koulen; né vers 1552, il était d'une vingtaine d'années plus jeune que sa sœur.

${ }^{4}$ O.O. 484,665 .

${ }^{5}$ O.O. 708 ; il aurait eu seize ans à sa mort.

${ }^{6} \mathrm{Je}$ résume ce que donnent à ce sujet Georg BecKer, Wilhelm Fabricius von Hilden, Wuppertal-Elberfeld, 1941 (70 p.), p. 9 s.; et deux mémoires d'Edouard Wiepen, Wilhelm Fabry von Hilden, dans les Beiträge zur Geschichte des Niederrheins, Düsseldorf 1910 et 1915. 
licher Haußfrawen weilandt des Ehrenthaften und Fürnemen Petri Andreae FabriciI, gewesenen Gerichtschreibers zu Hilden. Gehalten zu Hilden den 28. tag Aprilis, Anno 1612. durch H. Johannem KleE SleiDanum, Diener der Kirchen Christi daselbst».. C'est une brochure de 31 pages, datée MDCXII, mais sans lieu d'impression ni nom d'éditeur; la page du titre porte comme marque une image, Mundi Gloria, en médaillon ovale, qui se voit sur nombre de publications de FABRI. Ce sera lui plutôt que Sleidan qui aura pris l'initiative de l'impression.

Le sermon prend pour son texte 2 Cor. 5,1 et se divise en quatre points; le troisième (p.25-27) retiendra surtout notre attention car il est consacré à l'éloge des vertus de la défunte. Née de parents pieux, baptisée, élevée dans la crainte du Seigneur, elle a dès sa jeunesse écouté sa Parole et porté son joug. Dieu l'ayant appelée au mariage, elle connut quelques années de vie commune avec son «Haußwirt», dans l'affection et la paix. Jeune veuve elle se conforma à la doctrine de saint PAUL et se remaria; la mort impitoyable ayant encore ravi son second mari, elle en prit un troisième. Veuve pour la troisième fois, elle le resta dès lors définitivement pendant les vingt trois dernières années de sa vie. Elle éleva ses enfants, comme le veut saint PaUl, dans la crainte de Dieu, menant elle-même une vie sans reproche. Pour finir, Dieu, voulant la reprendre à lui, lui envoya une pénible maladie, qu'elle supporta patiemment, recommandant souvent son âme au Tout Puissant et confessant Jésus-Christ. - A quoi s'ajoute cette précision à tout le moins inattendue: Elle était si amie de la paix et de la bonne entente entre voisins, qu'au cours des quatre-vingts ans de sa vie terrestre, jamais elle ne fut convoquée devant la justice, ecclésiastique ou civile ... Les petits bourgeois de Hilden étaient-ils si querelleurs que ce fut un mérite spécial pour la veuve du greffier d'un de ces tribunaux de n'avoir pas passé elle-même en justice?

Voilà ce que raconte son gendre. Il nous donne ainsi une troisième variante du nom (AUF DER KULleN, AUF DEM SAND, zUM SAND); nous apprend qu'elle fut mariée trois fois, et non deux comme on le croyait jusqu'ici; que la mort de son troisième époux doit tomber vers 1589 , où elle avait environ 57 ans; que Gertgen a été son épouse à lui, Johann KLeE Sleidan ${ }^{8}$, et est bien vivante en 1612 .

' Il s'en trouve au moins deux exemplaires dans les bibliothèques publiques de Suisse, à Bâle (Universitätsbibliothek) et à St. Gall (Vadiana).

8 Tout ce qu'on savait de Gertgen jusqu'ici est qu'elle vivait encore en 1595: Wiepen (1915), 148 et note 36 . Elle avait alors environ vingt sept ans. - On veut espérer que les 
Ce n'est pas tout, cependant, car il y a une préface (p. 3-8), qui est plutôt une dédicace, et que Sleidde adresse, non à Guillaume FABRi, le membre illustre de la famille, mais à sa femme: Der Ehrentreichen, Tugentsamen und Gottseligen Frawen Mariae Colinaeteae, des Ehrentvesten und Hocherfarenen Wilhelmi Fabricir Hildani, lieben Hausfrawen, meiner vielgünstigen Schwegerin . . . Datée de Hilden. le 24 août 1612 et signée à nouveau Joannes Klee Sleidanus. On y lit à la fois l'éloge de la vie chrétienne modèle dont Colinetre a donné l'exemple lors de ses séjours à Hilden, du respect et de l'amour filial qu'elle témoignait à la défunte, toutes vertus dont le souvenir est resté vivant. Et celui du bon fils qu'a toujours été Guillaume, des soins dont il a voulu que sa mère fût entourée ${ }^{9}$; pour finir, c'est sur son ordre que la dépouille mortelle alla rejoindre celle de son premier mari et que leur épitaphe fut gravée sur une grande pierre. «Pour avoir honoré ses père et mère, je compte que Dieu le bénira, comme Il l'a fait jusqu'ici, et qu'il vivra longtemps»... .

Voilà pour dame FABRI, mère; et voici maintenant MARIE Colinet. Je ne rappelle pas les occasions nombreuses que saisit son mari de relever ses mérites; ni ce que WIEPEN a déjà recueilli sur son compte ${ }^{10}$. Ce que j'ai à y ajouter n'était pas absolument inconnu, car j'ai rencontré quelque part ${ }^{11}$ la mention qu'elle avait publié en 1638 un Alphabet nouveau et chrestien pour les jeunes apprentifs. Personne, en dehors des contemporains, y a-t-il jamais jeté les yeux ? Il est permis d'en douter. Pour ma part, sur la foi du titre et pensant à l'activité pratique de Madame FABRI aux côtés de son époux, au fait qu'à Payerne comme à Berne ils eurent souvent des élèves qui passèrent chez eux des mois, des années même, pour s'initier à l'art médical, je me figurais cet Alphabet comme un modeste recueil de conseils, à la fois maternels et techniques, à l'adresse de chirurgiens débutants. Combien la réalité est différente!

données nouvelles que nous apportons pourront faire l'object de recherches dans les archives locales; en particulier sur le pasteur J.K. SuEIDAN. Ce travail de paix redeviendra-t-il possible, et quand?

${ }^{9}$ De septembre 1611 à octobre 1612, FABRI, laissant les siens à Lausanne, est en Allemagne (avec un saut à Leyde); il y était venu, dit-il, voir ses amis (O.O., passim). Sans doute, l'état de santé de sa mère contribua à le retenir. Ce fut sa dernière visite à Hilden. MARIE CoLINET n'y est, je crois, pas revenue après 1595 .

${ }^{10}$ WIEPEN (1915), Index des personnes.

${ }^{11}$ Marie LipinsKa, Les femmes et le progrès des Sciences médicales, 1930, p. 73. 
C'est ce que dira d'emblée le titre, si nous le voyons au complet: «Alphabet nouveau et chrestien, pour les jeunes apprentifs, qui d'oresenavant commenceront d'aller en l'escole du S. Esprit, et ne scauroyent faillir d'estre sauvez, s'ils veulent ensuivre ce qui leur est ici descrit. - Vous y trouverez aussi la vie des Juifs et la nostre. - Le tout recueilli et assemblé du Vieil et Nouveau Testament, avec prières consolatoires pour l'âme affligée. - Par Marie Colinet, vefve de ce grand et vénérable Guil. Fabri de Hilden d'heureuse mémoire. - A Genève, Par Jean de la Fournaise MDCXXXVIII.» ... Et cet Alphabet, que j'avais eu la candeur de supposer une petite affaire, n'a pas moins de 824 pages! ${ }^{12}$

Commençons par constater que ce n'est pas le premier livre de dame FABRI; par sa Préface aux lecteurs nous apprenons l'existence d'«un petit Livre que j'avois fait au paravant, encore que quelques uns ayent pensé, et pensent encores, que je ne l'aye pas fait». Et ce qui achève de nous convaincre est le témoignage de deux amis, David Le Clerc, de Lausanne, intime des FABRI depuis plus de quarante ans, et un M.D.S.E. - Ministre du Saint Evangile - dont les initiales, M.B., n'ont jusqu'ici pas permis de fixer la personnalité ${ }^{13}$. Le premier rappelle le «petit et beau volume» où:

Vous nous avez monstré jadis

Comme ici bas chacun doit vivre, Pour des vertus le sentier suivre, Et entrer dans le Paradis.

Et le second précise que «Il y a quelques douze ans que me voulustes faire inspecteur de vostre premier livre intitulé: Le petit recueil de la Saincte Escriture. Et à présent avez aussi voulu me faire voir vostre second livre intitulé Alphabet nouveau»... Il est ainsi certain que vers $1621^{14}$ Dame FABRI fit ses débuts dans le monde littéraire.

Littérature d'édification, et non médicale. En quoi faisant, Marie ColiNET ne s'écartait point autant qu'on pourrait se le figurer des voies suivies par son époux; n'a-t-il pas, au millier de pages in folio de ses Opera Omnia, ajouté neuf ouvrages de format habituel, comptant à leur tour bien plus de mille pages, une bonne partie sous forme de cantiques et le surplus destiné

12 Bibl. publ. et universitaire, Genève; $56+768 \mathrm{p}$.

${ }^{13}$ Le Professeur H. MeYlan estime qu'il ne faisait pas partie du clergé vaudois.

${ }^{14}$ La lettre de M. B. est de 1633 au plus tard, car une note marginale, vers la fin, indique: «Monsieur FABRY n'estoit encore mort»; et le chirurgien est décédé en février 1634. Le Petit recueil ne se trouve dans aucune bibliothèque publique de Suisse. 
à guider la jeunesse sur le chemin étroit des vertus chrétiennes? De même qu'elle fut le modeste satellite du chirurgien, elle le reste donc de l'écrivain religieux. Non qu'elle le copie; c'est plus haut qu'elle cherche son inspiration; au moment où elle revendiquait sa qualité d'auteur du Petit recueil, elle ajoutait, pour ses deux écrits: «Ce neantmoins soyez asseurés que ... nul n'a esté l'auteur que Dieu, ny qui m'y aye assisté que le Sainct Esprit, qui m'a poussé à ce faire, et dicté maintes fois, dont j'estois moy mesme esmerveillée de ce qui me venoit au devant ... J'estois poussée, et comme portée à y adjouster toujours quelque chose, ... retenue par un doux et amiable advertissement de ne point quitter jusqu'à ce que j'eusse parachevé tout ce que j'y devois faire. Et vrayement, quand je fus venue jusques à la ligne qui fait ou est la dernière, je fus arrestée tout court, comme qui eust dit, Il suffit»...15

Au sentiment de l'auteur, l'Alphabet nouveau lui fut donc dicté par une muse, non l'une de celles qui faisaient cortège à l'Apollon paĩen, mais remplie de l'Esprit Saint. Muse qui se trouve tout naturellement au service du mouvement piétiste qui commençait à faire fermenter l'église et dont l'histoire, pour le Pays de Vaud, a été magistralement écrite par H. VUILLEUMIER $^{16}$. Il es fort intéressant de voir notre couple médical si intimément mêlé au petit groupe de zélateurs spirituels qui à cette époque se réclamaient du théologien d'Iéna, Johann Gerhard. Les Quinquaginta meditationes sacrae publiées par celui-ci en 1606 avaient aussitôt été jugées par FABRI le meilleur des livres après l'Ecriture sainte; et plus tard il n'avait eu de cesse avant d'obtenir d'un pasteur de ses amis qu'il les mît en français. Cela, avant tout, pour que sa «chérissime épouse», pût être la première à en jouir. Ce traducteur, qui fit sa carrière en Allemagne, pasteur à Nierstein près d'Oppenheim (rive gauche $\mathrm{du}$ Rhin, à peu près à mi-chemin entre Mannheim et Mayence), était du Pays de Vaud, Jean Combillon; son père, Pierre, avait été pasteur à Montet et Payerne (décédé en 1590). Parmi les pièces de vers qui accompagnent l'ouvrage s'en trouvent deux de son collègue Nicolas VIRET, alors pasteur à Curtilles; après avoir félicité FABRI de mettre ainsi les Cinquante Sainctes Meditations à la disposition des lecteurs de langue française, il s'adresse à dame $F_{A B R I}$ :

Combillon maria sa Gauloise faconde

Au grand Gerhard latin, MARIE, en ta faveur.

${ }^{15}$ Préface aux Lecteurs.

${ }^{16}$ Histoire de l'Eglise réformée du Pays de Vaud, III (1930), 183-550. 
Et toy vas estallant ce talent par le monde,

Afin de marier nostre ame à son Sauveur.

Viret avait même fait le projet de mettre ces Méditations en vers; on ne regrettera pas trop qu'il n'ait pas abouti. La Dédication est naturellement offerte par Combillon, à prudente et vertueuse Dame Marie Colinette; il lui «consacre de bon cœur ... ce petit livret ... Comme à celle pour laquelle il a esté traduit»... Il est bien puissant «pour toucher une ame devotieuse et l'induire à la contemplation des choses célestes» $\ldots{ }^{17}$ C'est dans la même atmosphère un peu particulière que se plaît encore un des ministres vaudois du cercle Fabri, Guillaume Dumont, pasteur à Montpreveyres, dont trois pièces accompagnent l'Alphabet nouveau; il y prend la défense de l'auteur contre les mondains qui pourraient s'aviser de trouver ... «estrange que la femme - Enseigne en la maison de Dieu»; bien au contraire,

Madame, de quelle couronne

Faudra-t-il que l'on vous couronne

Pour vos labeurs industrieux?

Une guirlande trimphante

Ne seroit pour vous suffisante

Car vous aurez celle des cieux.

Lui même, si la tradition est exacte, se proposait, tout comme VIRET, de mettre en vers les Méditations ${ }^{18}$.

Laissons de côté les pages terminales de ce gros volume, extraits des Méditations de J. GERHARD et pièces diverses, dont une empruntée au pasteur Dumoulin de Sedan; comment Marie Colinet a-t-elle compris sa tâche

${ }^{17}$ L'ouvrage de Combinlon, avec les textes latins et français en regard, Berne, Jacob Stuber, $1630,46 \mathrm{ff} .+723$ p.+ lf., est à la B.C.U., Lausanne, V 1356. Dans sa préface, en latin, FABRI nous apprend qu'une autre version française avait déjà paru en 1623, publiée à nouveau à Iena 1626, Cologne 1628, due à Simon Goulart, qui l'avait aussi dédiée à dame FABRI; double hommage dont sa modestie a bien pu souffrir quelque atteinte? En effet, Leonard Chester Jones, dans sa biographie de S. Goulart, Genève 1917, p. 647, mentionne la traduction de Goulart, dédiée à FABri et à dame Marie Colinet, sa femme. La Bibl. publ. de Genève en possède deux éditions, toutes deux de Genève, 1627, l'une (144 p.) publiée séparément, l'autre (326 p.) unie à la traduction d'un autre ouvrage de J. GERHARD.

18 VuILleumier, op. cit., III, 257. - L'Album studiosorum de L. Junod, II, signale N. VIreT à l'Académie de Lausanne en 1603 (No. 3019) et G. Dumont en 1614 (No. 3213), tandis que je n'y trouve pas Combillon, qui a pu y passer avant 1602 . 
et ordonné sa matière, ou, pour nous placer à son point de vue, qu'est-ce que l'Esprit Saint lui a dicté ? - Comme son titre le donnait à entendre, on peut reconnaître deux parties distinctes; l'une, un exposé des livres de la Bible, les suivant, assure l'auteur, du premier au dernier, de la création du monde (p. 1-38, la Genèse) à l'Apocalypse (p. 518-22), sur laquelle elle s'étend peu, en raison des mystères qui s'y trouvent et dont Dieu n'a pas voulu que nous eussions pleine connaissance. Après quoi elle passe à ce qui répond à la promesse du titre: "Vous y trouverez aussi la vie des Juifs et la nostre.» Elle est à ce moment si pressée d'obéir à son inspiration que rien ne nous avertit de l'entrée dans ce nouveau domaine, qu'un simple alinéa au milieu d'une page:

«Maintenant donc, dit-elle, que j'ai parlé de la vie des Juifs, et mesmes de ceux de devant le Deluge, ayant commencé dès Genèse jusques ici, et suivi tous les livres de suite du vieil et nouveau Testament, je fay une petite comparaison de leur vie avec la nostre et de leurs pechez avec les nostres, et aussi remarque les jugemens de Dieu prononcez à l'encontre d'iceux pechez; afin que quelques uns le lisans et sentans en leur conscience qu'ils sont entachés de quelcun des ditz pechez, s'amendent et repentent à ce qu'ils soyent sauvés.» «Petite comparaison», c'est à savoir, elle va de la p. 522 à la p. 702. La vieille dame ne voit pas en rose ce qui se passe sous ses yeux; et si les péchés des vieux Hébreux ne sont que trop manifestes, ceux des contemporains ne leur cèdent en rien. Le luxe de la toilette, en particulier, excite vivement son indignation ${ }^{19}$; des bonnets et coiffures jusqu'aux bas et souliers, le diable est partout à l'œuvre, ce «forgeur des pechez», si habile à séduire hommes et femmes. C'est lui qui a inspiré les extravagants et coûteux bonnets, chargés de «dorures, bordures, perles et pierreries, et par le derrière de la teste et par le dessus»; les «chevelures monstrueuses» qui défigurent hommes et femmes, et dont voici une description : «Il y en a [des femmes] qui allant par dehors accommodent leurs cheveux en sorte que quand le vent tire, ils volent par iceluy comme l'aile d'un rostissoir par la fumée dans une cheminée» . . . Et les souliers! Grâce au maître inventeur de péché, «avec une pommelle de bois qu'ils mettent sous le talon, ... si haute qu'elle leur fait lever le derrière du pied et le fait courber au milieu, qui fait qu'ils ne touchent la terre que du bout des arteils»... On le voit, ColinetTe, si elle revenait sur terre de nos jours, n'aurait rien à changer à cette partie de son réquisitoire; actuel au $20^{\mathrm{e}}$ siècle comme au $17^{\mathrm{e}}$, il risque

${ }^{19}$ Les extraits qui suivent, des p. 555, 562 s., $565,599$. 
de le rester toujours. Dans ce même ordre de choses, les excès du décolletage la froissent plus vivement encore: ces femmes, qui «vont la gorge descouverte jusques aux palettes des espaules, et toute la poitrine decouverte, et monstrent leurs tetins à chacun, ce qui est une honte. Jamais une femelle, craignante de Dieu, ne devroit laisser voir ses tetins qu'à un mari quand il l'auroit espousée; sinon que, comme nos corps sont en la main de Dieu et qu'il nous envoye quelque maladie cela va à part»... Bien loin de nourrir leurs enfants, on dirait qu'elles ne s'occupent de leurs «tetins» que pour les faire voir: «celles qui les ont gros, besongnent à l'entour pour les faire petits, et celles auxquels ils sont petits taschent à les faire croistre gros. Et les unes, toutes descouvertes entièrement; autres se mettent un fin linge par dessus avec des grandes dentelles qui leur vont par dessus la teste, passant par devant un œil et un tetin. Et toute ceste façon si lubrique et luxurieuse, que je m'esmerveille que nostre Dieu aye si grande patience»... Sommes nous en 1638 ou en 1938 ?

Autres péchés énormes et compositions diaboliques qui intéressent dame FABRI et sur quoi elle avait eu amplement l'occasion de recueillir des observations, au cours d'un demi siècle de pratique médicale: les superstitions, le recours aux sorciers, aux devins ${ }^{20}$. Bien souvent, nos maladies ne sont qu'un châtiment de Dieu pour nos fautes; mais au lieu de se reconnâtre coupables, beaucoup «se représentent avoir reçu quelque morceau venimeux; d'autres, leur avoir soufflé contre, d'autres, les avoir touchés en passant ...» «Quand il se voit quelcun qui soit possédé du malin, l'on court après, ... . luy demandans s'il ne sçait point qui a fait mourir, l'un ma fille, l'autre mon mari, ... l'autre, sa vache, l'autre, son cheval ... Desquelles choses le diable leur donne response selon leur désir, mais non selon vérité, car ce n'est pas sa nature»... Aux jeunes filles qui vont aux devins «pour sçavoir quel mari elles auront, ... ils enjoignent de mettre certaine chose sous ... le chevet ... de leur lict, puis devoir escouter la nuit ce qui leur sera dit»... Une fois le «monstre malin» installé chez ses victimes, il les «visitera, importun, avec grand bruit et autres incommoditez; et pour le deschasser se servent des enchanteurs ... Mais iceluy, fin et caut, fait semblant de se retirer, sçachant bien où les attendre».... «Autres ... font des singeries par les chemins et rues qui traversent en croix; en prenant des mesures qu'on appelle quarterons, et certaines herbes, faisant par ainsi maintes folies en croix, en barbotant certains charmes; . . . et le diable est

${ }^{20}$ P. 673-8. 
là, présent, qui s'en mocque.» - «Autres, pour avoir advis du dit diable, abusent des biens du Seigneur, ... en portant pain et argent en certains lieux où ceux qui le cerchent savent qu'il loge; et vont comme l'escrevice... Et ne disent mot ... quand ils vont le trouver, de peur qu'il ne les emporte»... «Quelle superstition et quel blasphème est-ce faire à Dieu, d'aller, pour guérir certaines maladies nommées à leur fantaisie, de bon matin en une estable à pourceaux, et devant le soleil levé - (mais c'est bien practiqué ce poinct, puisqu'ils ne sont pas dignes que ce beau flambeau les esclaire) où, ayans porté le malade, disent Nostre Pere, etc. Et après avoir rendu leur urine, font des signes de croix en marmottant l'on ne sçait quoy entre leurs dents, tapissant le dit estable de quelques herbes, selon leur invention; reitérant plusieurs fois ceste superstitieuse méthode»...

Nous connaissions Marie Colinet épouse, mère, chirurgienne, accoucheuse expérimentée. Sans doute ne verrons nous jamais le Petit recueil composé par elle. En revanche, grâce aux Sainctes Méditations dont la traduction lụi fut dédiée, aux témoignages des pasteurs et amis du cercle FABRI, grâce à son beaufrère JoHANn KLEe Sleidan, grâce surtout à ce qu'elle nous livre d'elle-même dans son Alphabet nouveau, le portrait intellectuel et moral que son mari a tracé d'elle se complète. Elle n'a pas été seulement bonne épouse et bonne mère, mais bonne bru, aussi. A l'égard du genre humain, elle est exigeante. Elle voudrait l'homme parfait et souffre de le voir si inférieur à son modèle, si peu désireux de s'y conformer. L'âge venu, elle ne peut s'empêcher de gronder des gens qui se conduisent si mal. Mais elle ne s'en tient pas là; ayant le bonheur de posséder le correctif à nos défaillances, la Parole divine, elle se sent pressée de le proposer à tous, d'encourager chacun à suivre ses préceptes. Nous n'avons pas de peine à nous représenter les époux FABRI, unis dans un même sentiment de reconnaissance et d'adoration envers Dieu, terminant leurs journées de travail assidu en faisant ensemble chaque soir leur prière. Ayant remercié le Tout Puissant de sa protection, ils se préparent à une nuit paisible en chantant quelques uns des cantiques composés par le bon chirurgien.

Le Mont, 24, novembre 1943 